\title{
A NOTE ON A CERTAIN CLASS OF FUNCTIONS RELATED TO HURWITZ ZETA FUNCTION AND LAMBERT TRANSFORM
}

\author{
R. K. RAINA AND T. S. NAHAR
}

\begin{abstract}
In this paper we obtain multiple-series generating relations involving a class of function $\theta_{\left(p_{n}\right)}^{\left(\mu_{n}\right)}\left(s, a ; x_{1}, \ldots, x_{n}\right)$ which are connected to the Hurwitz zeta function. Also, a new generalization of Lambert transform is introduced, and its relationship with the above class of functions further depicted.
\end{abstract}

\section{Introduction and Preliminaries}

The generalized (Hurwitz's) zeta function is defined by [3]

$$
\zeta(s, a)=\sum_{n=0}^{\infty}(a+n)^{-s}, \quad(\operatorname{Re}(s)>1 ; a \neq 0,-1, \ldots)
$$

and when $a=1$, we have

$$
\zeta(s, 1)=\sum_{n=1}^{\infty} n^{-s}=\zeta(s)
$$

where $\zeta(s)$ is the Riemann zeta function.

The function $\phi(x, s, a)([3, \mathrm{p} .27])$ extends (1.1) and is defined by

$$
\phi(x, s, a)=\sum_{n=0}^{\infty}(a+n)^{-s} x^{n} . \quad(\operatorname{Re}(a)>0 ;|x|<1)
$$

The integral representation of $\phi(x, s, a)$ is of form

$$
\phi(x, s, a)=\frac{1}{\Gamma(s)} \int_{0}^{\infty} t^{s-1} a^{-a t}\left(1-x e^{-t}\right)^{-1} d t
$$

provided that $R(a)>0$ (and either $|x| \leq 1, x \neq 1$, and $\operatorname{Re}(s)>0$, or $x=1$ and $\operatorname{Re}(s)>1)$.

Received February 25, 1999; revised April 26, 1999.

1991 Mathematics Subject Classification. 11M06, 11M35, 33C20.

Key words and phrases. Riemann zeta function, generalized Lauricella function, Laguerre Polynomials, Lambert transform, Mellin transform. 
We introduce a multivariable function $\theta_{\left(p_{n}\right)}^{\left(\mu_{n}\right)}\left(s, a ; x_{1}, \ldots, x_{n}\right)$ which is defined by

$$
\begin{aligned}
\theta_{\left(p_{n}\right)}^{\left(\mu_{n}\right)}\left(s, a ; x_{1}, \ldots, x_{n}\right) & =\theta_{\left(p_{1}, \ldots, p_{n}\right)}^{\left(\mu_{1}, \ldots, \mu_{n}\right)}\left(s, a ; x_{1}, \ldots, x_{n}\right) \\
& =\sum_{m_{1}, \ldots, m_{n}=0}^{\infty}(a+\Omega)^{-s} \prod_{i=1}^{n}\left\{\frac{\left(\mu_{i}\right)_{m_{i}}}{m_{i} !} x_{i}^{m_{i}}\right\}
\end{aligned}
$$

where $\Omega=\sum_{i=1}^{n} p_{i} m_{i}, \operatorname{Re}(a)>0, \mu_{i} \geq 1$ (either $\left|x_{i}\right|<1, x_{i} \neq 1$; or $\left|x_{i}\right|=1, \operatorname{Re}(s)>n$, $\forall i=1, \ldots, n)$.

Equivalently, the integral representation of $\theta_{\left(p_{n}\right)}^{\left(\mu_{n}\right)}\left(s, a ; x_{1}, \ldots, x_{n}\right)$ is given by

$$
\theta_{\left(p_{n}\right)}^{\left(\mu_{n}\right)}\left(s, a ; x_{1}, \ldots, x_{n}\right)=\frac{1}{\Gamma(s)} \int_{0}^{\infty} t^{s-1} e^{-a t} \prod_{i=1}^{n}\left(1-x_{i} e^{-p_{i} t}\right)^{-\mu_{i}} d t
$$

provided that $\operatorname{Re}\left(\mu_{i}\right) \geq 1, \operatorname{Re}\left(p_{i}\right)>0 ;(i=1, \ldots, n), \operatorname{Re}(a)>0$ (and either $\max \left\{\left|x_{i}\right|\right\} \leq$ $1, x_{i} \neq 1(i=1, \ldots, n)$, and $\operatorname{Re}(s)>0$; or $x_{i}=1(i=1, \ldots, n)$ and $\left.\operatorname{Re}(s)>\sum_{i=1}^{n} \mu_{i}\right)$. As usual the symbol $(\lambda)_{n}$ stands for

$$
(\lambda)_{n}=\left\{\begin{array}{ll}
1, & \text { if } n=0 \\
\lambda(\lambda+1) \cdots(\lambda+n-1), & \text { if } n \in \mathbb{N}
\end{array} \quad(\lambda \neq 0,-1,-2, \ldots)\right.
$$

\section{Special cases of (1.5)}

(i) When $n=p=1$, we have

$$
\phi_{1}^{\mu}(s, a ; x)=\sum_{m=0}^{\infty}(a+m)^{-s} \frac{(\mu)_{m} x^{m}}{m !}=\phi_{\mu}^{*}(x, s, a) .
$$

The function $\phi_{\mu}^{*}(x, s, a)$ was studied recently by Goyal and Laddha [4].

(ii) For $n=p=\mu=1$, we have

$$
\theta_{1}^{1}(s, a ; 1)=\sum_{m=0}^{\infty}(a+m)^{-s} x^{m}=\phi(x, s, a)
$$

Evidently, the Hurwitz's zeta function (1.1) is given by the relation

$$
\theta_{1}^{1}(s, a ; 1)=\zeta(s, a) .
$$

(iii) Corresponding to $\mu_{i}=x_{i}=1(\forall i=1, \ldots, n)$, we have

$$
\theta_{p_{1}, \ldots, p_{n}}^{1, \ldots, 1}(s, a ; 1, \ldots, 1)=\sum_{m_{1}, \ldots, m_{n}=0}^{\infty}(a+\Omega)^{-s}=\zeta_{n}\left(s, a ; p_{1}, \ldots, p_{n}\right),
$$

where $\Omega=\sum_{i=1}^{n} p_{i} m_{i}$. The class of functions $\zeta_{n}\left(s, a ; p_{1}, \ldots, p_{n}\right)$ is the $n$-tuple Hurwitz $\zeta$-function introduced by Barnes [1] (see also [9]). 
(iv) For $\mu_{i}=p_{i}=x_{i}=1(\forall i=1, \ldots, n)$, we have

$$
\theta_{1, \ldots, 1}^{1, \ldots, 1}(s, a ; 1, \ldots, 1)=\sum_{m_{1}, \ldots, m_{n}=0}^{\infty}\left(a+\Omega^{*}\right)^{-s}=\zeta_{n}(s, a),
$$

where $\Omega^{*}=\sum_{i=1}^{n} m_{i}$. The function $\zeta_{n}(s, a)$ is the multiple Hurwitz's zeta function (studied recently by Choi [2]).

In the present paper we first obtain certain multiple-series generating functions involving the multivariable function $\theta_{\left(p_{n}\right)}^{\left(\mu_{n}\right)}\left(s, a ; x_{1}, \ldots, x_{n}\right)$ defined by (1.5) above. A new generalization of Lambert transform is introduced, and its inversion formula, and relationship with the function $\theta_{\left(p_{n}\right)}^{\left(\mu_{n}\right)}\left(s, a ; x_{1}, \ldots, x_{n}\right)$ are also pointed out. The results presented provide extensions to some of the results in [4] and [6].

\section{Generating Relations}

Using (1.5) and the multinomial expansion ([7, p.329])

$$
\sum_{k_{1}, \ldots, k_{r}=0}^{\infty}(\lambda)_{\sum_{i=1}^{r} k_{i}} \prod_{i=1}^{r}\left\{\frac{x_{i}^{k_{i}}}{k_{i} !}\right\}=\left(1-\sum_{i=1}^{r} x_{i}\right)^{-\lambda}
$$

provided that $\left|\sum_{i=1}^{r} x_{i}\right|<1$, we easily obtain the generating function:

$\sum_{k_{1}, \ldots, k_{r}=0}^{\infty}(\lambda)_{\sum_{i=1}^{r} k_{i}} \theta_{\left(p_{n}\right)}^{\left(\mu_{n}\right)}\left(\lambda+\sum_{i=1}^{r} k_{i}, a ; x_{1}, \ldots, x_{n}\right) \prod_{i=1}^{r}\left\{\frac{t_{i}^{k_{i}}}{k_{i} !}\right\}=\theta_{\left(p_{n}\right)}^{\left(\mu_{n}\right)}\left(\lambda, a-\sum_{i=1}^{r} t_{i} ; x_{1}, \ldots, x_{n}\right)$,

provided that $\left|\sum_{i=1}^{r} t_{i}\right|<|a|, \lambda \neq 1$, and $\left|x_{i}\right|<1(i=1, \ldots, n)$.

The generating function (2.2) admits of a further extension. Indeed, in terms of the Lauricella's multiple hypergeometric series $F_{D}^{r}$ ([7, p.33]), which is defined by

$$
\begin{aligned}
& F_{D}^{(r)}\left[a, b_{1}, \ldots, b_{r} ; c ; x_{1}, \ldots, x_{r}\right] \\
= & \sum_{m_{1}, \ldots, m_{r}=0}^{\infty} \frac{(a)_{m_{1}+\cdots+m_{r}}\left(b_{1}\right)_{m_{1}} \cdots\left(b_{r}\right)_{m_{r}}}{(c)_{m_{1}+\cdots+m_{r}}} \prod_{i=1}^{r}\left\{\frac{x_{i}^{m_{i}}}{m_{i} !}\right\},\left(\max \left\{\left|x_{1}\right|, \ldots,\left|x_{r}\right|\right\}<1\right)
\end{aligned}
$$

it follows from (1.5) that

$$
\begin{aligned}
& \sum_{k_{1}, \ldots, k_{r}=0}^{\infty} \frac{\sum_{i=1}^{(\lambda)} k_{i}}{(\nu) \sum_{i=1}^{r} k_{i}} \prod_{i=1}^{r}\left\{\frac{\left(\mu_{i}\right)_{k_{i}} t_{i}^{k_{i}}}{k_{i} !}\right\} \theta_{\left(p_{n}\right)}^{\left(\mu_{n}\right)}\left(\lambda+\sum_{i=1}^{r}\left(\mu_{i}+k_{i}\right)-\nu, a ; x_{1}, \ldots, x_{n}\right) \\
= & \sum_{m_{1}, \ldots, m_{n}=0}^{\infty}(a+\Omega)^{-\lambda+\nu-\sum_{i=1}^{r} \mu_{i} n} \prod_{i=1}^{n}\left\{\frac{\left(\mu_{i}\right)_{m_{i}} x_{i}^{m_{i}}}{m_{i} !}\right\} F_{D}^{(r)}\left[\lambda, \mu_{1}, \ldots, \mu_{r} ; \nu ; \frac{t_{1}}{a+\Omega}, \ldots, \frac{t_{r}}{a+\Omega}\right]
\end{aligned}
$$


provided that $\max \left\{\left|\frac{t_{i}}{a}\right|\right\}<1, \mu_{i} \geq 1, \forall i=1, \ldots, r ; \operatorname{Re}\left(\lambda+\sum_{i=1}^{r} \mu_{i}\right)>\operatorname{Re}(v)>0$. More generally, (2.4) may further be extended in the form

$$
\begin{aligned}
& \sum_{k_{1}, \ldots, k_{r}=0}^{\infty} \nabla\left(k_{1}, \ldots, k_{r}\right) \theta_{\left(p_{n}\right)}^{\left(\mu_{n}\right)}\left(\sigma+\sum_{i=1}^{r} k_{i}, a ; x_{1}, \ldots, x_{n}\right) \prod_{i=1}^{r}\left\{\frac{t_{i}^{k_{i}}}{k_{1} !}\right\} \\
= & \sum_{m_{1}, \ldots, m_{n}=0}^{\infty}(a+\Omega)^{-\sigma} \prod_{i=1}^{n}\left\{\frac{\left(\mu_{i}\right)_{m_{i}} x_{i}^{m_{i}}}{m_{i} !}\right\} F_{l: s_{1} ; \ldots ; s_{r}}^{P: Q_{1} \ldots ; Q_{r}}\left[\frac{t_{1}}{a+\Omega}, \ldots, \frac{t_{r}}{a+\Omega}\right],
\end{aligned}
$$

provided that

(i) $1+\sum_{i=1}^{r}\left(s_{i}-Q_{i}\right)+l-P \geq 0$ and either $P>l$ and $\sum_{i=1}^{r}\left|\frac{t_{i}}{a}\right|^{\frac{1}{P-l}}$ or $P \leq l$ and $\max \left\{\left|\frac{t i=1}{a}\right|\right\}<1(i=1, \ldots, r)$

(ii) $\mu_{i} \geq 1, \operatorname{Re}(a)>0$

where

$$
\nabla\left(k_{1}, \ldots, k_{r}\right)=\frac{\prod_{i=1}^{P}\left(a_{i}\right) \sum_{i=1}^{r} k_{i} \prod_{i=1}^{Q_{1}}\left(b_{i}^{1}\right)_{k_{1}} \cdots \prod_{i=1}^{Q_{r}}\left(b_{i}^{(r)}\right)_{k_{r}}}{\prod_{i=1}^{l}\left(\alpha_{i}\right)_{\sum_{i=1}^{r} k_{i}} \prod_{i=1}^{s_{1}}\left(\beta_{i}^{1}\right)_{k_{1}} \cdots \prod_{i=1}^{s_{r}}\left(\beta_{i}^{(r)}\right)_{k_{r}}} .
$$

The function $F_{l: s_{1} ; \ldots ; i_{r}}^{P: Q_{1} ; \ldots ; Q_{r}}\left[x_{1}, \ldots, x_{r}\right]$ occurring in $(2.5)$ is the generalized Lauicella series in several variables defined as follows (see $[7, \mathrm{p} .38]$ ):

$$
\begin{aligned}
F_{l: s_{1} ; \ldots ; s_{r}}^{P: Q_{1} ; \ldots, Q_{r}}\left[x_{1}, \ldots, x_{r}\right] & =F_{l: s_{1} ; \ldots ; s_{r}}^{P: Q_{1} ; \ldots ; Q_{r}}\left[\begin{array}{l}
\left(a_{P}\right):\left(b_{Q_{1}}^{1}\right) ; \ldots ;\left(b_{Q_{r}}^{(r)}\right) ; \\
\left(\alpha_{l}\right):\left(\beta_{s_{1}}^{1}\right) ; \ldots ;\left(\beta_{s_{r}}^{(r)}\right) ;
\end{array} x_{1}, \ldots, x_{r}\right] \\
& =\sum_{k_{1}, \ldots, k_{r}=0}^{\infty} \nabla\left(k_{1}, \ldots, k_{r}\right) \prod_{i=1}^{r}\left\{\frac{x_{i}^{k_{i}}}{k_{i} !}\right\}
\end{aligned}
$$

where, $\nabla\left(k_{1}, \ldots, k_{r}\right)$ is defined above by $(2.6)$.

Next, consider a set of polynomials $\left\{S_{m}^{q}(x)\right\}_{m=0}^{\infty}$ defined by [5, p.1, Eq. (1)]:

$$
S_{m}^{q}(x)=\sum_{j=0}^{[m / q]} \frac{(-m)_{q j}}{j !} C(j) x^{j}, \quad\left(q \in \mathbb{N} ; m \in \mathbb{N}_{0}=\mathbb{N} U\{0\}\right)
$$

where $C(j)$ are arbitrary constants (real or complex).

Then, by simple series rearrangement method, and applying (2.2) in the process, we are lead to the following multiple generating relation:

$$
\begin{aligned}
& \sum_{k_{1}, \ldots, k_{r}=0}^{\infty}\left(\lambda \sum_{i=1}^{r} k_{i} \theta_{\left(p_{n}\right)}^{\left(\mu_{n}\right)}\left(\lambda+\sum_{i=1}^{r} k_{i}, a ; x_{1}, \ldots, x_{n}\right) \prod_{i=1}^{r}\left\{S_{k_{i}}^{q_{i}}\left(y_{i}\right) \frac{t_{i}^{k_{i}}}{k_{i} !}\right\}\right. \\
= & \sum_{k_{1}, \ldots, k_{r}=0}^{\infty}(\lambda)_{\sum_{i=1}^{r} k_{i} q_{i}} \theta_{\left(p_{n}\right)}^{\left(\mu_{n}\right)}\left(\lambda+\sum_{i=1}^{r} k_{i} q_{i}, a-\sum_{i=1}^{r} t_{i} ; x_{1}, \ldots, x_{n}\right) \prod_{i=1}^{r}\left\{\frac{C\left(k_{i}\right)\left\{y_{i}\left(-t_{i}\right)^{q_{i}}\right\}^{k_{i}}}{k_{i} !}\right\} .
\end{aligned}
$$


By specializing the sequence $C\left(k_{i}\right)$ as follows:

$$
C\left(k_{i}\right)=\frac{\prod_{j=1}^{P_{i}}\left(a_{j}^{(i)}\right)_{k_{i}}}{q_{i}^{k_{i} q_{i}} \prod_{j=1}^{Q_{i}}\left(b_{j}^{(i)}\right)_{k_{i}}},
$$

we then find from (2.9) that

$$
\begin{aligned}
& \sum_{k_{1}, \ldots, k_{r}=0}^{\infty}(\lambda) \sum_{i=1}^{r} k_{i} \theta_{\left(p_{n}\right)}^{\left(\mu_{n}\right)}\left(\lambda+\sum_{i=1}^{r} k_{i}, a ; x_{1}, \ldots, x_{n}\right) \prod_{i=1}^{r}\left\{q_{i}+P_{i} F_{Q_{i}}\left[\begin{array}{l}
\Delta\left(q_{i},-k_{i}\right),\left(a_{P_{i}}^{(i)}\right) ; y_{i} \\
\left(b_{Q_{i}}^{i}\right)
\end{array}\right]\right. \\
= & \sum_{k_{1}, \ldots, k_{r}=0}^{\infty}(\lambda)_{i}^{k_{i}} k_{i} ! \\
\sum_{i=1}^{r} k_{i} q_{i} & \theta_{\left(p_{n}\right)}^{\left(\mu_{n}\right)}\left(\lambda+\sum_{i=1}^{r} k_{i} q_{i}, a-\sum_{i=1}^{r} t_{i} ; x_{1}, \ldots, x_{n}\right) \\
& \prod_{i=1}^{r}\left\{\frac{\prod_{j=1}^{P_{i}}\left(a_{j}^{(i)}\right)_{k_{i}}}{k_{i} ! \prod_{j=1}^{Q_{i}}\left(b_{j}^{(i)}\right)_{k_{i}}}\left\{y_{i}\left(\frac{-t_{i}}{q_{i}}\right)^{q_{i}}\right\}^{k_{i}}\right\}
\end{aligned}
$$

where $\Delta(m, \lambda)$ denotes the array of $m$-parameters

$$
\frac{\lambda}{m}, \frac{\lambda+1}{m}, \ldots, \frac{\lambda+m-1}{m} \quad(m \in \mathbb{N}) .
$$

Example. By involving the Lagueree polynomials (which occurs when $P_{i}=0$, $\left.Q_{i}=1=q_{i}, b_{1}^{(i)}=1+\alpha_{i}(i=1, \ldots, r)\right),(2.11)$ yields

$$
\begin{aligned}
& \sum_{k_{1}, \ldots, k_{r}=0}^{\infty}(\lambda) \sum_{i=1}^{r} k_{i} \theta_{\left(p_{n}\right)}^{\left(\mu_{n}\right)}\left(\lambda+\sum_{i=1}^{r} k_{i}, a ; x_{1}, \ldots, x_{n}\right) \prod_{i=1}^{r}\left\{\frac{L_{k_{i}}^{\alpha_{i}}\left(y_{i}\right) t_{i}^{k_{i}}}{\left(1+\alpha_{i}\right)_{k_{i}}}\right\} \\
= & \sum_{k_{1}, \ldots, k_{r}=0}^{\infty}(\lambda)_{\sum_{i=1}^{r} k_{i}} \theta_{\left(p_{n}\right)}^{\left(\mu_{n}\right)}\left(\lambda+\sum_{i=1}^{r} k_{i}, a-\sum_{i=1}^{r} t_{i} ; x_{1}, \ldots, x_{n}\right) \prod_{i=1}^{r}\left\{\frac{\left(-y_{i} t_{i}\right)^{k_{i}}}{\left(1+\alpha_{i}\right)_{k_{i}}}\right\} .
\end{aligned}
$$

Several other examples similar to $(2.12)$ can be obtained from (2.11) by suitably specializing the sequence $C\left(k_{i}\right)$. We omit further details.

\section{An Integral Transform}

Let $f(t)(t \geq 0)$ be a continuous function, and

$$
f(t)=O\left(e^{k t}\right)(t \rightarrow \infty)
$$


Then, the Lambert transform of $f(t)$ is defined by

$$
F(s)=L M\{f(t)\}=\int_{0}^{\infty} \frac{s t}{e^{s t}-1} f(t) d t . \quad(\operatorname{Re}(s)>0)
$$

We introduce a generalization of the Lambert transform (3.2) in the following form:

$$
\begin{aligned}
H^{*}\{f(t)\} & =H_{\left(p_{n}\right)}^{\left(\mu_{n}\right)}\left(x_{1}, \ldots, x_{n} ; s\right)=H_{\left(p_{1}, \ldots, p_{n}\right)}^{\left(\mu_{1}, \ldots, \mu_{n}\right)}\left(x_{1}, \ldots, x_{n} ; s\right) \\
& =\int_{0}^{\infty} \frac{s t}{\prod_{i=1}^{n}\left(e^{p_{i} s t}-x_{i}\right)^{\mu_{i}}} f(t) d t
\end{aligned}
$$

provided that $\operatorname{Re}(s)>0, p_{i}>0,\left|\mu_{i}\right| \geq 1, \max \left|x_{i}\right| \leq 1(\forall i=1, \ldots, n), f(t) \in A$ and $\operatorname{Re}(\gamma)>-2$, where $A$ denotes the class of functions $f(t)$ which are continuous for $t>0$ and satisfy the order estimates:

$$
f(t)= \begin{cases}O\left(t^{\gamma}\right) & \left(t \rightarrow 0_{+}\right) \\ O\left(t^{\delta}\right) & (t \rightarrow \infty)\end{cases}
$$

The parameter $\delta$ is unrestricated, in general, since $\operatorname{Re}(s)>0, p_{i}>0(i=1, \ldots, n)$. We note that on putting $n=p=1$, and setting $f=t^{k-1} g$ in (3.3), we have

$$
H_{1}^{\mu}(x ; s)=\int_{0}^{\infty} \frac{s t^{k}}{\left(e^{s t}-x\right)^{\mu}} g(t) d t
$$

which was recently studied by Goyal and Laddha [4]. Evidently,

$$
H_{1}^{1}(x ; s)=\int_{0}^{\infty} \frac{s t}{\left(e^{s t}-x\right)} f(t) d t
$$

the transform investigated by Raina and Srivastava [6]. It readily follows from (3.3) and (1.6) that

$$
H^{*}\left\{t^{\alpha-1} e^{-\nu s t}\right\}=\frac{\Gamma(\alpha+1)}{s^{\alpha}} \theta_{\left(p_{n}\right)}^{\left(\mu_{n}\right)}\left(\alpha+1, \nu+\sum_{i=1}^{n} p_{i} \mu_{i} ; x_{1}, \ldots, x_{n}\right),
$$

provided that $\operatorname{Re}(s)>0, \operatorname{Re}(\alpha)>-1, \operatorname{Re}(\nu)>0, \mu_{i} \geq 1, p_{i}>0$, and $\left|x_{i}\right| \leq 1$ $(\forall i=1, \ldots, n)$.

Further, in view of (2.8) and (3.7) we obtain

$$
\begin{aligned}
& H^{*}\left\{t^{\alpha-1} e^{-\nu s t} \prod_{i=1}^{r}\left(S_{m_{i}}^{q_{i}}\left(y_{i} t\right)\right)\right\} \\
= & \frac{1}{s^{\alpha}} \sum_{j_{1}=0}^{\left[m_{1} / q_{1}\right]} \cdots \sum_{j_{r}=0}^{\left[m_{r} / q_{r}\right]} \Gamma\left(1+\alpha+\sum_{i=1}^{r} j_{i}\right) \prod_{i=1}^{r}\left\{\frac{\left(-m_{i}\right) q_{i} j_{i}}{j_{i} !} C\left(j_{i}\right)\left(\frac{y_{i}}{s}\right)^{j_{i}}\right\} \\
& \cdot \theta_{\left(p_{n}\right)}^{\left(\mu_{n}\right)}\left(1+\alpha+\sum_{i=1}^{r} j_{i}, \nu+\sum_{i=1}^{n} p_{i} \mu_{i} ; x_{1}, \ldots, x_{n}\right)
\end{aligned}
$$


provided that $\operatorname{Re}(s)>0, \operatorname{Re}(\alpha)>-1, \operatorname{Re}(\nu)>0$ and $\mu_{i} \geq 1, p_{i}>0,\left|x_{i}\right| \leq 1(\forall i=$ $1, \ldots, n)$ and $q_{i} \in \mathbb{N}, m_{i} \in \mathbb{N}_{0}(\forall i=1, \ldots, r)$. In particular, when $C\left(j_{i}\right)=\frac{\left(1+\alpha_{i}\right)_{m_{i}}}{\left(1+\alpha_{i}\right)_{j_{i}}}$, $q_{i}=1(\forall i=1, \ldots, r)$, then $(3.8)$ in terms of Laguerre polynomials gives

$$
\begin{aligned}
& H^{*}\left\{t^{\alpha-1} e^{-\nu s t} L_{m_{1}}^{\alpha_{1}}\left(x_{1} t\right) \cdots L_{m_{r}}^{\alpha_{r}}\left(x_{r} t\right)\right\} \\
= & \prod_{i=1}^{r}\left\{\frac{\Gamma\left(m_{i}+\alpha_{i}+1\right)}{m_{i} !}\right\} s^{-\alpha} \sum_{j_{1}=0}^{m_{1}} \cdots \sum_{j_{r}=0}^{m_{r}} \Gamma\left(1+\alpha+\sum_{i=1}^{r} j_{i}\right) \\
& \cdot \theta_{\left(p_{n}\right)}^{\left(\mu_{n}\right)}\left(1+\alpha+\sum_{i=1}^{r} j_{i}, \nu+\sum_{i=1}^{n} p_{i} \mu_{i} ; x_{1}, \ldots, x_{n}\right) \prod_{i=1}^{r}\left\{\frac{\left(-m_{i}\right)_{j_{i}}\left(\frac{x_{i}}{s}\right)^{j_{i}}}{\Gamma\left(1+\alpha_{i}+j_{i}\right) j_{i} !}\right\} .
\end{aligned}
$$

\section{Inversion formula for the transform (3.3)}

Applying the Mellin transform [8, p.46], (3.3) then gives

$$
\begin{aligned}
\Psi(k) & =\int_{0}^{\infty} s^{-k-1} H_{\left(p_{n}\right)}^{\left(\mu_{n}\right)}\left(x_{1}, \ldots, x_{n} ; s\right) d s \\
& =\int_{0}^{\infty} s^{-k-1}\left\{\int_{0}^{\infty} s t \prod_{i=1}^{n}\left(e^{p_{i} s t}-x_{i}\right)^{-\mu_{i}} f(t) d t\right\} d s \\
& =\int_{0}^{\infty} t f(t)\left\{\int_{0}^{\infty} s^{-k} \prod_{i=1}^{n}\left(e^{p_{i} s t}-x_{i}\right)^{-\mu_{i}} d s\right\} d t, \\
& =\Gamma(1-k) \theta_{\left(p_{n}\right)}^{\left(\mu_{n}\right)}\left(1-k, \sum_{i=1}^{n} p_{i} \mu_{i} ; x_{1}, \ldots, x_{n}\right) \int_{0}^{\infty} t^{k} f(t) d t,
\end{aligned}
$$

provided that, in addition to the existence and convergence conditions stated with (3.3), we also require that $\operatorname{Re}(k)<1$, for the convergence of the inner $s$-integral in (3.10) above.

By the Mellin inversion theorem [8, p.46], we obtain the following inversion formula for the integral transform (3.3):

$$
\begin{aligned}
& \frac{1}{2}[f(t+0)+f(t-0)] \\
= & \frac{1}{2 \pi i} \int_{\sigma-i \infty}^{\sigma+i \infty}\left\{\Gamma(1-k) \theta_{\left(p_{n}\right)}^{\left(\mu_{n}\right)}\left(1-k, \sum_{i=1}^{n} p_{i} \mu_{i} ; x_{1}, \ldots, x_{n}\right)\right\}^{-1} t^{-k-1} \Psi(k) d k,
\end{aligned}
$$

provided that $\sigma>1 / 2, \operatorname{Re}(k)<1, t^{k} f(t) \in L(0, \infty), f(t)$ is of bounded variation in the neighbourhood of the point $t, \Psi(k)$ is given by (3.10), and $\mu_{i} \geq 1, p_{i}>0, \max \left\{\left|x_{i}\right|\right\} \leq 1$ $(\forall i=1, \ldots, n)$. 


\section{Acknowledgement}

Authors are thankful to the referee for suggestions. The work of the second author was supported by the university Grants Commission, under Grant No. F. 4-5 (10)/97/(MRP/CRO).

\section{References}

[1] E. W. Barnes, On the theory of the multiple gamma function, Philos. Trans. Roy. Soc., 199(1904), 374-439.

[2] J. Choi, Multiple gamma function and their applications, in Proc. Internat. Conf. on Analysis (editied by Y.C. Kim), Yeungnam Univeristy, Korea (1996), 73-84.

[3] A. Erdélyi, W. Magnus, F. Oberhettinger and F. G. Tricomi, Higher Transcendental Functions, Vol. 1, McGraw-Hill, New York, Toronto and Londom, 1953.

[4] S. P. Goyal and R. K. Laddha, On the generalized Riemann zeta function and the generalized Lambert transform, Ganita Sandesh, 11(1997), 99-108.

[5] H. M. Srivastava, A contour integral involving Fox's H-function, Indian J. Math., 14(1972), 1-6.

[6] R. K. Raina and H. M. Srivastava, Certain results associated with the generalized Riemann zeta functions, Rev. Tec. Ing. Univ. Zulia, 18(1995), 301-304.

[7] H. M. Srivastava and P. W. Karlson, Multiple Gaussian Hypergeometric Series, John Wiley and Sons, New York, 1984.

[8] E. C. Titchmarsh, Introduction to the Theory of Fourier Integrals, Clarendon Press, Oxford, 1948.

[9] E. T. Whittaker and G. N. Watson, A Course of Modern Analysis (4th ed.), Cambridge University Press, 1963.

Department of Mathematics, C.T.A.E. Campus, Udaipur Udaipur - 313001, Rajasthan, India.

Department of Mathematics, Govt. Postgraduate College, Bhilwara, Bhilwara - 311001, Rajasthan, India. 